\section{Ultimate imaging system for early detection}

For the ultimate technology to accelerate minimally invasive, preventive dentistry, come and see the CALCIVIS imaging system at the BDIA Dental Showcase 2018 at ExCel London on 4-6 October 2018.

The science behind this innovative device was inspired by the bioluminescent (light emitting) mechanism that is seen in nature by marine animals, such as jellyfish.

CALCIVIS applies a highly specific bioluminescent photoprotein, which in the presence of free calcium ions released from actively demineralising tooth surfaces, produces a low level light. An integrated intraoral sensor then detects this light and displays a precise, glowing map of active demineralisation at the chair side.

The CALCIVIS imaging system is an early detection device that enables practitioners to identify demineralisation at its earliest and most reversible stage and to plan prompt management to prevent dental caries and erosion.

With clear, engaging images, the CALCIVIS imaging system is also an advanced communication tool. Dental professionals can explain demineralisation, illustrate problem areas and motivate

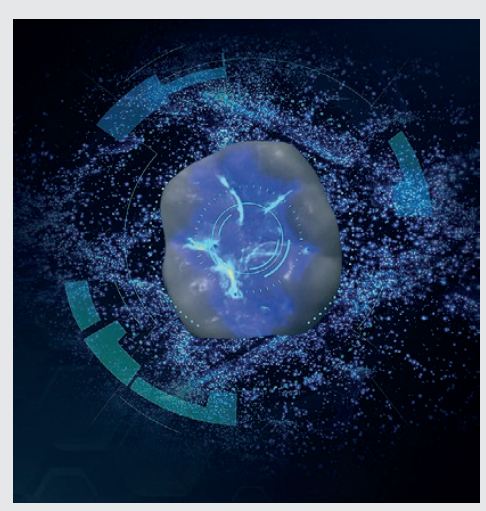
patients by helping them to understand and improve their oral health. The CALCIVIS team will be on Stand D51 to show delegates the imaging system.

More information is available at www.calcivis. com, or by calling 0131 6585152 or emailing info@calcivis.com.

\section{Latest power toothbrush technology}

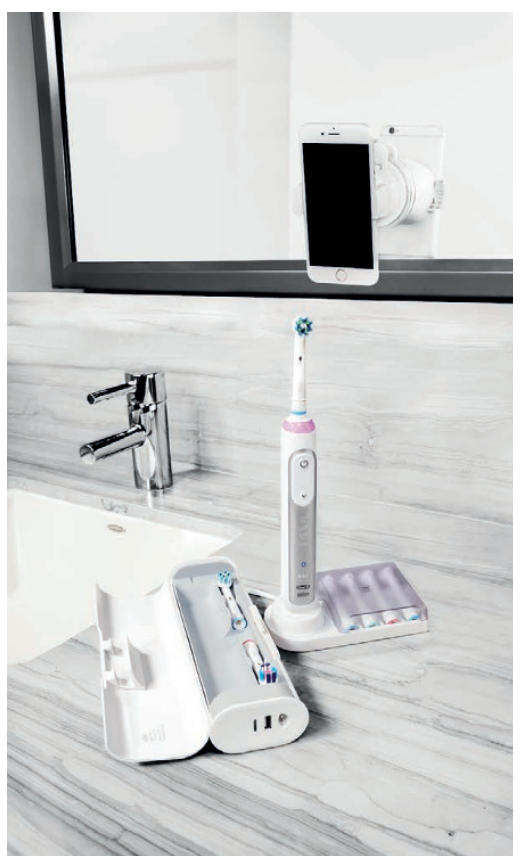

Delegates at the BDIA

Dental Showcase 2018

should visit Oral-B at

Stand I50 to see for

themselves the company's

flagship power tooth-

brush - Oral-B

GENIUS 9000.

By combining built-in motion sensor technology and video recognition using a smartphone's camera with the Oral-B app, all zones of the user's mouth can be tracked, providing real-time feedback on which areas they've brushed and which areas have been missed.

The mechanical

benefits of Oral-B's power toothbrushes compliment the chemical efficacy afforded by their toothpaste, says the company.

Its Pro-Expert toothpaste includes stabilised Stannous Fluoride which provides 24 -hour oral protection and those wanting more specific protection, might prefer Oral-B Gum \& Enamel Repair toothpaste, which actively protects gums and strengthens enamel.

Visitors will also be able to view Oral-B's new 'Strong Teeth Make Strong Kids' campaign, which this year will provide up to 20,000 dental professionals with educational materials on how to support parents with their kids dental care needs.

Oral-B will also be running free 30-minute CPD sessions on their stand (Stand I50), focusing on children's oral health.

\title{
Bringing the demo to the customer
}

Can't make it to BDIA Dental Showcase 2018? Well, PlanDemo can come to you.

The Planmeca company is inviting conference attendees to visit it on Stand I26 at the BDIA Dental Showcase 2018 where they will be able to get a hands-on experience with the Planmeca range of equipment.

Its product specialists will be there to demonstrate the products, from the world-class ProMax 3D CBCT with pioneering Ultra Low Dose protocol and new CALM (Correction Algorithm for Latent Movement), through to the Compact Classic dental unit.

Delegates will also have the option to explore the simplicity of the company's PlanFIT system, combining its lightweight intraoral scanner; Emerald and PlanMill 40 S milling unit. If practitioners cannot make it to Dental Showcase to learn more about the Planmeca portfolio, the company can bring its mobile experience centre; PlanDemo to practitioners.

Onboard is the high-tech Compact iTouch dental unit featuring world-class integration and infection control, fully-functioning ProMax 3D with ProFace, advanced intraoral X-ray, ProX and the company's full chair side digital impression solution PlanFIT.

More information is available by calling 08005200330 or visiting www.plandemo.co.uk.dentalelite.co.uk or calling 01788545900 .

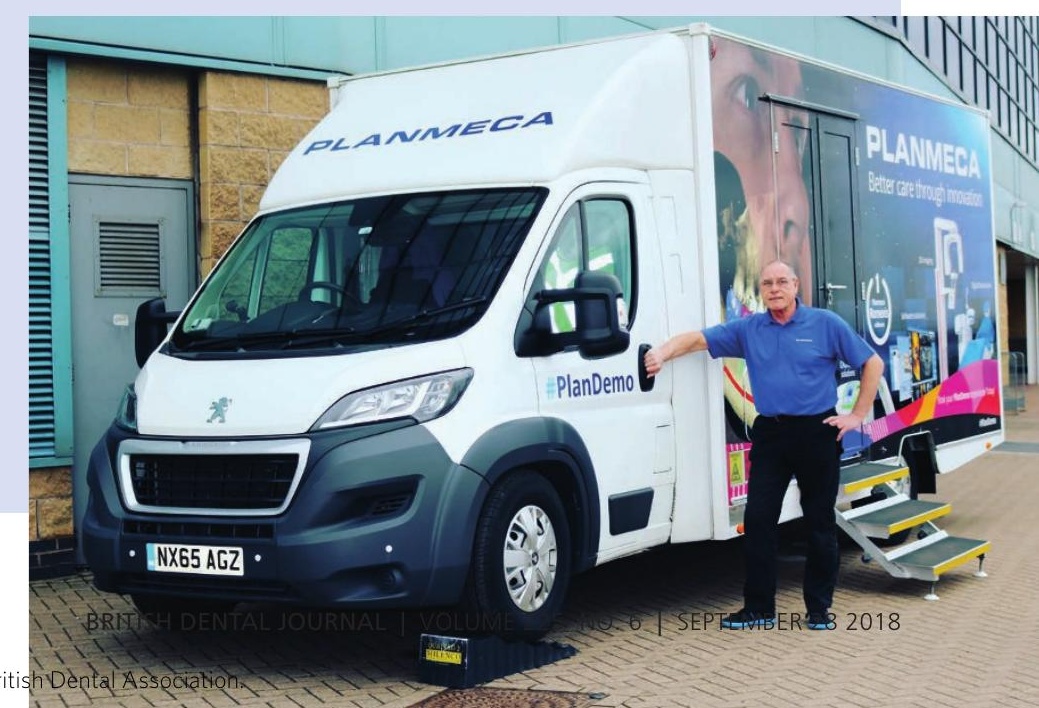

